\title{
Towards System-Mediated Car Passenger Communication
}

\author{
Angela Mahr, Margarita Pentcheva, Christian Müller \\ German Research Center for Artificial Intelligence (DFKI) \\ Building D32, Campus, Stuhlsatzenhausweg 3 \\ 66123 Saarbrücken, Germany \\ \{Firstname.lastname@dfki.de\}
}

\begin{abstract}
In this paper, we outline a system that supports the communication between passengers by transmitting speech (and maybe also video) of the communication partners back and forth. A study is presented that addresses the questions: 1. Is listening to noisy speech coming from the backseat really distracting the driver? Subjects are rating the truth of common-sense statements played from the back of the car (clear, noisy) while driving with a drive simulator. NOISY is rated significantly more distracting than CLEAR while objective driving performance only degrades for men but not for women.
\end{abstract}

\section{Categories and Subject Descriptors}

H.5.2 [Information interfaces and presentation]: User Interfaces, User-centered design

\section{General Terms}

DESIGN,HUMAN FACTORS

\section{Keywords}

infotainment, automotive, multi-party, lane change

\section{INTRODUCTION}

Research on automotive assistance and infotainment systems has traditionally focussed on one person: the driver. However, even with commuting to work, an average of $10.2 \%$ chose to carpool in the US in 2004, as a study by American Community Survey revealed [1]. With leisure time traveling, the number of cars with more than one passenger can be expected to be much higher. Hence, taking passengers into account when designing in-car systems is a reasonable thing to do. In our research, we lay the foundations for a new generation of context-aware multimodal interfaces for car passengers that support the interaction of the passengers with the car, the interaction of the passengers and the road environment as well as the interaction between the passengers, mediated by the system. This study contributes to the latter aspect.

Copyright held by author(s)

AutomotiveUI'09, September 21-22, 2009, Essen, Germany ACM 978-1-60558-571-0/09/009

\section{DESIGN SKETCH}

When taking multiple users into account, the problem of appropriate alignment of multimodal output presentation has to be addressed. To this end, appropriate contents need to be routed to output devices bound to specific seat areas but also adapted to the role of the speaker. Drivers might, for example, decide that their children should just have access to some specific areas of the infotainment system like for example for watching DVD or playing games. Thus, a multimodal dialog platform must support role-specific access to the underlying services and appropriate configuration possibilities. Due to the requirement of minimal distraction, the way of presenting information to the driver is especially crucial. Hence, we propose mainly auditive output: The driver interacts mainly using speech and auditive output; the co-driver has an additional small screen that can - under certain conditions - be shared with the driver. The passengers on the backseat have access to full blown entertainment touch screens. For technical details regarding the microphone technique one might have a look at the DVE system recently available in the VW Multivan [5].

Thinking on how to support interaction (communication) between passengers, the most immediate problem appears to be that conversations between people in the front and people in the back are difficult: due to driving noise, the acoustic characteristics of the car as well as the fact that everyone faces one direction, it is hard to understand what is being said. One straightforward way to support the communication with such a setup would be to transmit speech (and maybe also video) of the communication partners back and forth. With the right equipment, this could function in the same way as noise canceling headphones, which cancel the ambient noise and thereby enhance speech. The study presented here addresses the question: Is listening to noisy speech coming from the backseat really distracting the driver? And would this conversational task during driving affect men and women to the same degree?

\section{EXPERIMENT}

We recorded 63 yes/no common-sense statements that were either true or false like for example "In Norway a scarf and gloves are useful winter clothings", "At the beach you should always take care of snowslides". Out of these sentences two lists with equal proportions of true and false sentences were made. Half of the recordings were overlaid with car noise (NOISY), the other half was not preprocessed (CLEAR). Between subjects the choice of list that was to be presented clearly was balanced. The sentences were played from the 
Table 1: Subjective rating by the subjects how demanding the task was.

\begin{tabular}{llll} 
& CLEAR & NOISY & sign. diff \\
\hline hard to listen & 1.88 & 2.75 & $\mathrm{p}<0.01$ \\
distracted me from driving & 2.75 & 3.83 & $\mathrm{p}<0.01$ \\
compromised my driving & 2.88 & 3.63 & $\mathrm{p}<0.05$
\end{tabular}

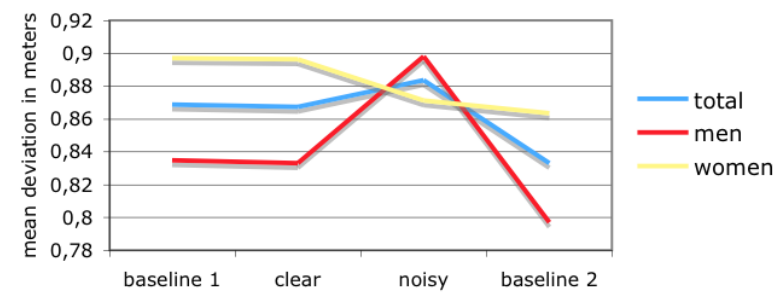

Figure 1: Objective measure of distraction using lane change task.

backseat while the subject was driving (with a drive simulation software) in order to assess the level of distraction. The subjects were instructed to say whether the statement is true or false. In addition to the objective measure, we asked the subject to rate the subjective distraction after each condition. Prior to the experiment, a motivating example was provided to the subjects: "Imagine that you, as a driver, have a conversation with the passengers in the back seat. It is loud in your car (vehicle/motor or ambient noises, radio) and you have to make great efforts to hear your partner." After the experiment, we asked the subjects whether they would appreciate a system like that. We measured the driver distraction using the standardized "lane change task" (LCT) [3], a simple laboratory dual-task method that is intended to estimate driver distraction (ISO Draft International Standard 26022). We used the following statement for the subjective rating of distraction: 1 . The communication task distracted me from driving; 2 . The communication task compromised my driving performance; 3. I found it hard to listen to the presented questions. The possible scale of answers was from 1 ("I do not agree") to 5 ("I fully agree").

24 subjects (11 men and 13 women), were paid to participate in a user study. The age range was between 21 and 60 with an average age of 35.9 years for men and 33.2 years for women. The entire experiment took about one hour to complete. However, a significant part of that time (appr. $30 \mathrm{~min}$ ) was designated to a different study (not presented here). The recordings were played from the back by another experimenter. After warmup and first baseline, the main part of the experiment started. The order of the (CLEAR and NOISY) conditions was balanced between subjects and gender. A second baseline was measured afterwards, followed by the other part of the experiment (different study).

Table 1 shows the results of the subjective rating. NOISY was rated demanding, distracting from driving, and compromising driving performance. The differences between CLEAR and NOISY were statistically significant for each of the questions $(p<0.01$ resp. $p<0.05)$. Figure 1 shows the mean deviation in meters between a normative model and the actual driving in LCT. A repeated measures ANOVA was carried out with the relevant covariates age (significant correlation with driving performance in the baselines, $r=.4, p<.05$ ) and order of the experimental conditions (due to expected learning effects): The main effect for condition was not significant, $F(3,60)=1.16$, ns. as well as the main effect for gender, $F(1,20)=1.61$, ns. As expected the main effect of age was significant $F(1,20)=15.32, p<.01$. The interaction between gender and condition was not significant over all conditions either $F(3,60)=1.83$, ns. But as indicated earlier the central questions are, whether drivers were distracted by the CLEAR or the NOISY condition. In order to test this, orthogonal contrasts were conducted. The first comparison of the two baselines was not significant $F(1,20)$ $<1, n s$. Then the NOISY condition was contrasted with the CLEAR condition and we found no significant difference $F(1,20)=2.14$, ns. As a last contrast we compared both baselines with both speech conditions, but didn't find a significant difference either $F(1,20)=1.98, n s$.

A probable explanation for not having found the expected and subjectively rated stronger interference of the NOISY condition with the driving task than for the CLEAR condition might be the lack of sensitivity of the lane change task. One more suggestion we have had was that there might be an interaction of condition and gender concerning the NOISY condition, so we conducted a contrast for the interaction comparing the NOISY condition with the CLEAR condition. We found a significant interaction for this contrast, $F(1,20)$ $=5.59, p<.05$. This made us have a closer look at each gender separately and the same contrasts were repeated for each of the groups. For men the comparison of the NOISY condition with all other conditions was significant $F(1,10)$ $=5.21, p<.05$, but for women it was not $F(1,12)<1$, ns. This means, that performance degrades in the NOISY condition only for men but not for women. The analysis of the subjective rating regarding the gender aspect revealed no significant interaction, $F(1,22)=2.35, p=.14$, but a similar pattern.

\section{CONCLUSIONS}

We outlined a system that supports the communication between passengers by transmitting speech (and maybe also video) of the communication partners back and forth. We reported results of a study indicating that listening to noisy speech from the back is distracting drivers (subjective rating), especially men (subjective rating and drive simulator).

\section{REFERENCES}

[1] American community survey 2004: Percent of workers who traveled to work by carpool. http://www.statemaster.com/. 09/05/27.

[2] M. Burmester, R. Graf, J. Hellbrück, and M. Ansgar. Usability - Der Mensch im Fahrzeug. In Infotainmentsysteme im Kraftfahrzeug. Springer, 2008.

[3] S. Mattes. The lane change task as a tool for driver distraction evaluation. In H. Strasser, H. Rausch, and H. Bubb, editors, Quality of Work and Products in Enterprises of the Future. Ergonomia, 2003.

[4] C. Müller-Bagehl and P. Endt, editors. Infotainment/Telematik im Fahrzeug - Trends für die Serienentwicklung. Haus der Technik Fachbuch 38. Expert, Renningen, 2004.

[5] S. Steenhagen. The first automotive implementation of a digital voice enhancement system, 2005. (09/07/31) http://www.appliedsignalprocessing.com/dve2.htm. 